\title{
PREMIXED FLAME RESPONSE TO HELICAL DISTURBANCES: MEAN FLAME NON-AXISYMMETRY EFFECTS*
}

\author{
Vishal Acharya ${ }^{\mathrm{a}}$, Tim Lieuwen ${ }^{\mathrm{a}}$ \\ ${ }^{a}$ School of Aerospace Engineering \\ Georgia Institute of Technology \\ Atlanta, GA, USA 30332-0150 \\ Corresponding author: Vishal Acharya \\ Ben T. Zinn Aerospace Combustion Laboratory \\ School of Aerospace Engineering \\ Georgia Institute of Technology \\ 635 Strong St NW, Atlanta, GA, USA 30318 \\ Email: vishal.sa@gatech.edu \\ Phone: 404-944-3647
}

Fax: 404-385-7117

\footnotetext{
*Expanded from conference paper GT2014-27059 titled "Response of Non-axisymmetric Premixed, Swirl Flames to Helical Disturbances" presented at the ASME IGTI Turbo Expo 2014 in Dusseldorf, Germany

(C) 2015. This manuscript version is made available under the Elsevier user license http://www.elsevier.com/open-access/userlicense/1.0/
} 


\section{Abstract}

A key component of the thermoacoustic instability feedback mechanism is the excitation of hydrodynamic flow disturbances by narrowband acoustic fluctuations. In earlier work, we considered the response of time-averaged axisymmetric flames to helical disturbances. That study showed that although all helical modes cause local flame wrinkling, only the axisymmetric hydrodynamic mode, $m=0$, contributes to the global heat release rate fluctuations. This paper extends this work to consider time-averaged flames that are non-axisymmetric. We show that distortions of the unforced flame shape changes its receptivity to helical disturbances, as it does not allow for the perfect destructive interference seen in axisymmetric flames. This results in a non-zero global flame response contribution from helical modes. Given that helical modes often have the largest amplitudes in jet flows, particularly those with swirl, these results show that the degree of non axisymmetry of the flame has an important influence in determining which hydrodynamic modes, axisymmetric or helical, control the global heat release response of the flame. These points have been illustrated with example calculations that show how different control parameters and the degree of time-averaged non-axisymmetry influence the global flame response. An important implication of these results relates to scaling results from simplified geometries where a round jet is placed inside a round enclosure, to more realistic ones (such as where multiple jets are placed next to each other) where the flame shape will be distorted from being perfectly circular.

Keywords: premixed flame; swirl-stabilized; non-axisymmetric; helical disturbances; level-set. 


\section{Nomenclature}

$A \quad=$ Spatially integrated ("global") flame area

$\hat{B}_{i, m} \quad=$ Helical mode amplitude for mode $m$ of velocity component in the $i$-direction

$G \quad=$ Level set function (or) iso-contour variable for flame surface

$L_{f} \quad=$ Characteristic flame length scale (flame height)

$R \quad=$ Non-dimensional center-body radius

St $=$ Strouhal number, ratio of characteristic axial time-scale and acoustic time-scale

$U_{0} \quad=$ Characteristic velocity scale

$U_{c} \quad=$ Axial phase speed of the velocity disturbance

$\vec{e}_{i} \quad=$ unit vector along $i$ coordinate direction

$k_{c}=$ Non-dimensional phase speed of velocity disturbance, $=U_{c} / U_{0}$

$s_{L} \quad=$ Laminar flame speed

$\vec{u} \quad=$ Velocity vector

$\varepsilon \quad=$ Ratio of amplitude of velocity disturbance and characteristic mean velocity scale

$\eta \quad=$ Degree of azimuthal variation in mean quantities

$\sigma \quad=$ Swirl parameter, $=\Omega / \omega$

$\omega \quad=$ Acoustic forcing frequency

$\xi \quad=$ Vertical flame location (measured along axial direction)

$\psi \quad=$ Vertical half angle of the stationary flame (w.r.t. axial direction)

$\Omega \quad=$ Characteristic azimuthal frequency (or) angular rate of swirl for solid body rotation

()$_{r, \theta, z, x}=$ Radial, azimuthal, axial and transverse x-direction component, respectively 


$$
\begin{aligned}
& ()_{t, n}=\text { Tangential and normal components to the mean flame surface, respectively } \\
& ()^{*} \quad=\text { Dimensional quantity } \\
& ()^{\prime} \quad=\text { Fluctuating component } \\
& \overline{(~} \quad=\text { Mean component } \\
& () \quad=\text { Frequency domain representation of corresponding time domain quantity }
\end{aligned}
$$




\section{Introduction}

Oscillations due to combustion instability are an important concern in modern combustors due to their degrading effects on system performance and hardware life. At the heart of this mechanism is the strong coupling between acoustics, flow hydrodynamics and the unsteady heat release rate oscillations. [1-3]. Of specific focus in this paper is the dynamics of premixed swirling flames subjected to helical flow fluctuations. While recent experimental research work has expanded the literature on this phenomena, there exists a need for deeper understanding of the flame response mechanism when hydrodynamic flow instabilities such as the helical modes of a swirling flow are in play [4-9].

The hydrodynamic instability of flows that are swirl and shear dominated manifests itself in several different unsteady flow phenomena, of which the precessing vortex core and helical shear layers are very important [10-14]. In the presence of sufficient swirl, the global instability of the flow results in a large vortex breakdown [11, 15-17]. These complex flow features are of importance in a combustor configurations since they control many different operational characteristics of the combustor such as flame stabilization, blow-off limits, flame shape and thermoacoustic instability [18].

This paper focuses on the sensitivity of premixed flames to harmonic flow fluctuations. It follows the results from several prior studies that modeled the dynamics of premixed flames subjected to direct acoustic and vortical flow fluctuations $[19,20]$. These dynamics were modeled using the level-set equation to analyze both the space-time distribution of unsteady flame surface position fluctuations as well as the spatially integrated global heat release rate fluctuations [20-22]. In all of these studies, either a two-dimensional or axisymmetric framework 
was considered, thus restricting the model to only symmetric flow fluctuations or features, such as excited ring vortices. In the case of both swirling and non-swirling jets there exist strong helical flow fluctuations that are inherently non-axisymmetric For example, Figure 1 reproduces a snapshot from the level-set-LES simulation by Huang and Yang [23]. Notice the asymmetry in vortices that are shed off from the separating shear layer, indicating the strong presence of helical modes in the flow disturbances.

In order to describe the non-axisymmetric nature of the flow fluctuations, we can decompose their azimuthal variation into azimuthal modes or helical modes. This modal decomposition results in a complete set of basis functions that are orthogonal to each other:

$$
\begin{aligned}
& \hat{u}_{i}^{\prime}(r, \theta, z, \omega)=\sum_{m=-\infty}^{\infty} \hat{B}_{i, m}(r, z, \omega) e^{i m \theta} \\
& \hat{B}_{i, m}(r, z, \omega)=\frac{1}{2 \pi} \int_{0}^{2 \pi} e^{-i m \theta} \hat{u}_{i}^{\prime}(r, \theta, z, \omega) d \theta
\end{aligned}
$$

where $\hat{u}_{i}^{\prime}$ is the Fourier transform of the fluctuating velocity field in the $i$-direction and $\hat{B}_{i, m}$ is the amplitude of its helical mode number $m$. Note that $m=0$ is the axisymmetric mode, while $m<0$ and $m>0$ are the non-axisymmetric modes that are co-rotating and counter-rotating with respect to the positive swirl direction.

As mentioned before, helical flow disturbances are present in both swirling and non-swirling round jet and wake flows. For non-swirling jets, isothermal stability analysis has shown that the separating boundary layer thickness near the jet exit controls the strength of the axisymmetric $m=0$ mode relative to the low order helical modes but helical modes dominate further downstream of the potential core [24]. Similarly, the dominant instability mode in non-swirling, axisymmetric wakes is the helical, $m= \pm 1$ modes [25]. The stability of non-swirling jets has also been shown to be sensitive to confinement [26]. 
In the case of swirling flows, the bias in the direction of swirl has a great influence on hydrodynamic stability by biasing the strength of co-rotating and counter-rotating helical modes [27-31]. For example, stability calculations were performed by Loiseleux et al. [27], for a Rankine vortex model with the following velocity profiles:

$$
\begin{aligned}
& r<R: \bar{U}_{z}(r)=U_{\infty}+\Delta U, \quad \bar{U}_{r}(r)=0, \quad \bar{U}_{\theta}(r)=\Omega r \\
& r>R: \bar{U}_{z}(r)=U_{\infty}, \quad \bar{U}_{r}(r)=0, \quad \bar{U}_{\theta}(r)=\Omega R^{2} / r
\end{aligned}
$$

Their results indicate that increasing swirl number decreases the temporal instability growth rate of the axisymmetric, $m=0$ mode (presumably because the flow profile is centrifugally stable $[32,33])$. Impacts of swirl on helical modes are more complex, generally showing nonmonotonic behavior with increases in swirl.

In addition to these intrinsic hydrodynamic instabilities of swirling flows, confined combustion chambers also exhibit natural acoustic oscillations. These acoustic oscillations interact strongly with the hydrodynamic flow instabilities. Imaging and measurements of acoustically forced premixed swirling flames have shown the strong presence of the influences of helical flow fluctuations along the flame location $[2,34]$. The presence of a swirling component in the mean flow causes wrinkles to be propagated along the flame in both the axial and azimuthal direction [35-38]. Note that due to the bias in the swirl direction, helical modes that are winding co- and counter- to the swirl direction interact differently with wrinkle propagation. Recent experimental work by Moeck et al. [4] and Stohr et al. [5] clearly show the azimuthally rotating heat release oscillations on the flame that are excited by helical disturbances. In addition, recent experiments by Worth and Dawson [6] analyzed the global heat release dynamics due to self-excited circumferential instabilities in an annular combustor. For annular combustors, depending upon the azimuthal location of the nozzle in the standing wave, they would excite different helical disturbances and thus differing flame response $[6,8]$. The 
flames excited by helical disturbances, as opposed to axisymmetric structures, had much smaller amplitude of heat release oscillations.

The Flame Transfer function $(F T F)$, defined as the ratio of the spatially integrated heat release to a reference velocity perturbation has been used extensively to characterize the global flame response of premixed flame. In earlier work, the authors showed that this quantity was zero for all helical modes $(m \neq 0)$ and that only the $m=0$ mode contributed to the FTF when the timeaveraged mean flame was axisymmetric [39]. In other words, although helical, $m \neq 0$, modes may cause significant local space-time wrinkling of the flame, they lead to zero fluctuations in the spatially integrated heat release, due to phase cancellation in the azimuthal direction.

The axisymmetric assumption on the nominal flame shape and flow field is a useful one for understanding the dynamics of a single reacting jet/wake flow in a circular geometry. However, a single flame becomes non-axisymmetric when placed in a non-circular geometry, such as a combustor with a square or sector cross-section. In addition, multiple nozzle configurations generally lead to strong flame-flame interactions, whose shapes are not axisymmetric [6]. An illustrative example of this point is shown in Figure 2, showing a side view and end-on visualization of a 5-flame configuration housed in a circular combustor (see Samarsinghe et al. [40] for details of experiment). Note the nearly "squircle" (square with rounded edges) crosssection of the central flame. Computational work by Staffelbach et al. [41] as well as experimental work by Bourgouin et al. [3] and Worth and Dawson [6, 7, 42] in an annular combustor also clearly show the change in shape due to flame-flame interactions. The sensitivity of the flame to helical modes is fundamentally different in axisymmetric or non-axisymmetric environments, as shown later in this paper.

The overall flame response question is further complicated in instances where the magnitude 
of helical modes are much stronger than that of axisymmetric modes. For example, an example plot from a transversely forced experiment is shown in Figure 3[8]. This plot clearly shows that during the presence of transverse acoustic excitation, the $m= \pm 1$ mode is an order of magnitude stronger than the axisymmetric mode. This raises the question of whether the response of weakly non-axisymmetric flames is dominated by the higher flame sensitivity to the weaker $m=0$ mode, or the weak flame sensitivity to the high amplitude helical modes.

Expanding on the earlier study by the authors [39] that analyzed the sensitivity of axisymmetric flames to disturbances, this paper focuses on cases where the time-averaged flame/flow is non-axisymmetric. This introduces an important new degree of freedom to the physics. Apart from the fundamental physical insight to be gained, this work also has important practical implications on how to scale thermoacoustic stability results from single nozzle or sector combustors to full scale systems. The paper is organized to first detail the analytical formulation, and to then apply this to examples with explicit calculations and solutions. In these examples, the sensitivity of the spatially integrated heat release to helical modes is quantified using the Flame Transfer Function $(F T F)$.

\section{Analytical Formulation}

This section detailed the analytical formulation using a front tracking approach for thin premixed flames [43-47] The flame is assumed to be located at the zero iso-contour of an implicit function denoted as, $G(r, \theta, z, t)$. The evolution of this contour is tracked using the $G$-equation [44, 47].

$$
\frac{\partial G}{\partial t^{*}}+\vec{u}^{*} \cdot \vec{\nabla} G=s_{L}^{*}|\vec{\nabla} G|
$$


Where $\vec{u}$ is the flow velocity at the flame front and the superscript " "*" denotes a dimensional variable.

For the purpose of an analytically tractable analysis, the following assumptions are made: (i) the flame is an infinitely thin interface that divides reactants and products, (ii) the flame position is a function of a single spatial coordinate, i.e. it is not multi-valued, (iii) negligible density jump across the flame and (iv) the flame speed, $s_{L}$, is constant. The motivation and implications of these assumptions and their applicability are detailed in Acharya et al. [39]. Using assumption (ii), the implicit function $G(r, \theta, z, t)$ is converted to an explicit flame position using: $G=z^{*}-\xi^{*}(r, \theta, t)$. Define the non-dimensional scheme:

$$
\begin{aligned}
& t=t^{*} /\left(L_{f} / U_{0}\right) \\
& u=u^{*} / U_{0} \quad s_{L}=s_{L}^{*} / U_{0} \\
& r=r^{*} / L_{f} \quad z=z^{*} / L_{f} \quad \xi=\xi^{*} / L_{f}
\end{aligned}
$$

Here, $L_{f}$ is a characteristic flame length scale (flame height), and $U_{0}$ is a velocity scale (representative of the spatial flow field). Rewriting Eq.(3) in cylindrical polar coordinates:

$$
\frac{\partial \xi}{\partial t}+u_{r} \frac{\partial \xi}{\partial r}+\frac{u_{\theta}}{r} \frac{\partial \xi}{\partial \theta}+s_{L}\left[\left(\frac{\partial \xi}{\partial r}\right)^{2}+\frac{1}{r^{2}}\left(\frac{\partial \xi}{\partial \theta}\right)^{2}+1\right]^{1 / 2}=u_{z}
$$

The flame configuration for this analysis shown in Figure 4 consists of a center-body stabilized premixed flame in a swirling flow. A representative time averaged flame position is shown by the dashed curves, and the perturbed flame by the solid curves. The time-averaged vertical flame angle is locally denoted as $\psi$. The center-body stabilization implies a fixed boundary condition for $\xi$ : 


$$
\left.\xi(r=R, \theta, t)\right|_{\text {flame-holder }}=0
$$

The flame position and velocity field can be decomposed into a time-averaged steady mean (overbar notation) and a spatio-temporally varying disturbance (superscript prime notation):

$$
\begin{aligned}
& \xi(r, \theta, t)=\bar{\xi}(r, \theta)+\varepsilon \xi^{\prime}(r, \theta, t) \\
& u_{i}(r, \theta, t)=\bar{U}_{i}(r, \theta)+\varepsilon u_{i}^{\prime}(r, \theta, t)
\end{aligned}
$$

Where the sub-script $i$ denotes the $r, \theta$, or $z$ coordinate. Here, $\varepsilon$ is a non-dimensional small parameter denoting the magnitude of the disturbances. Thus the evolution equations for the timeaveraged flame position and its linear perturbation are [48, 49]:

$$
\begin{aligned}
& \bar{U}_{r} \frac{\partial \bar{\xi}}{\partial r}+\frac{\bar{U}_{\theta}}{r} \frac{\partial \bar{\xi}}{\partial \theta}=\bar{U}_{z}-s_{L}\left[\left(\frac{\partial \bar{\xi}}{\partial r}\right)^{2}+\left(\frac{1}{r} \frac{\partial \bar{\xi}}{\partial \theta}\right)^{2}+1\right]^{1 / 2} \\
& \frac{\partial \xi^{\prime}}{\partial t}+\overrightarrow{\vec{u}}_{t} \cdot \vec{\nabla} \xi^{\prime}=\left(u_{z}^{\prime}-u_{r}^{\prime} \cot \psi-u_{\theta}^{\prime} \frac{1}{r} \frac{\partial \bar{\xi}}{\partial \theta}\right)
\end{aligned}
$$

where

$$
\frac{\partial \bar{\xi}}{\partial r}=\cot \psi(r, \theta)
$$

The net tangential velocity vector along the flame surface, due to both the azimuthal and axial velocity components, is given by:

$$
\overrightarrow{\vec{u}}_{t}=\overrightarrow{\vec{U}}-s_{L} \vec{e}_{n}
$$

Here $\vec{e}_{n}$ is the local unit normal vector that points from the time averaged flame surface into the products. The frequency domain representation of Eq. (8) is

$$
i 2 \pi S t \hat{\xi}^{\prime}+\overrightarrow{\bar{u}}_{t} \cdot \vec{\nabla} \hat{\xi}^{\prime}=\left(\hat{u}_{z}^{\prime}-\hat{u}_{r}^{\prime} \frac{\partial \bar{\xi}}{\partial r}-\hat{u}_{\theta}^{\prime} \frac{1}{r} \frac{\partial \bar{\xi}}{\partial \theta}\right)
$$


Where the 'overhats' denote corresponding quantities in frequency domain. The solution to this equation, can also decomposed into its modes as:

$$
\hat{\xi}^{\prime}(r, \theta, S t)=\sum_{m} \hat{\xi}_{j}^{\prime}(r, S t) e^{i m \theta}
$$

Note that even within this linear framework, helical modes associated with the fluctuating flow-field do not necessarily translate to a corresponding azimuthal disturbance in the fluctuating flame response for a general non-axisymmetric mean flow/flame, i.e. in general, a mode $m$ in the flow field leads to a mode $j \neq m$ for the flame wrinkling. For example, in a nonaxisymmetric flame, an axisymmetric disturbance, $m=0$, excites non-axisymmetric flame wrinkles. This point is explained in detail using example calculations presented later.

\section{Spatially Integrated Global Flame Response}

For acoustically compact sound sources (e.g., flames), the spatial distribution of the source is not important; rather, it is the spatially integrated "global" heat release, $Q^{\prime}(t)$ that controls the sound emissions from the flame and/or thermoacoustic stability of the system [1, 20, 21, 43]. We shall further consider the global flame response in the rest of the paper. The unsteady heat release per unit area of the premixed flame surface is given by $q=\rho s_{L} h_{R}$, where $\rho$ is the density of unburnt gases, $s_{L}$ is the laminar consumption speed and $h_{R}$ is the heat of reaction. Thus, in a flame consuming homogeneous reactants and with a constant burning velocity, $s_{L}$, the local heat release is directly proportional to the local flame surface area. Stretch sensitive flames that are wrinkled also introduce additional heat release fluctuations through flame speed oscillations [50]. This effect becomes significant when the radius of wrinkling and/or scale of the velocity gradient is of the same order of magnitude as the Markstein length and is not considered further in this 
study. The differential surface area is given by:

$$
d A=r d \theta d r \sqrt{1+\left(\frac{\partial \xi}{\partial r}\right)^{2}+\left(\frac{1}{r} \frac{\partial \xi}{\partial \theta}\right)^{2}}
$$

The global heat release for constant burning velocity flames is directly proportional to the global flame surface area and thus, the spatially integrated total flame surface area is given by,

$$
A=\iint_{r, \theta} r d \theta d r \sqrt{1+\left(\frac{\partial \xi}{\partial r}\right)^{2}+\left(\frac{1}{r} \frac{\partial \xi}{\partial \theta}\right)^{2}}
$$

We can linearize this equation, thus decomposing it into the time-averaged flame area and the fluctuating flame area (in the frequency domain) as:

$$
\begin{aligned}
& \bar{A}=\iint_{r, \theta} r d r d \theta \sqrt{1+\left(\bar{\xi}_{, r}\right)^{2}+\left(\bar{\xi}_{, \theta} / r\right)^{2}} \\
& \hat{A}^{\prime}=\iint_{r, \theta}\left\{r d \theta d r \frac{\left(\bar{\xi}_{, r} \hat{\xi}_{, r}^{\prime}+\frac{1}{r^{2}} \bar{\xi}_{, \theta} \hat{\xi}_{, \theta}^{\prime}\right)}{\sqrt{1+\left(\bar{\xi}_{, r}\right)^{2}+\left(\bar{\xi}_{, \theta} / r\right)^{2}}}\right\}
\end{aligned}
$$

Here $\xi_{, r}$ and $\xi_{, \theta}$ denote derivatives with respect to $r$ and $\theta$ respectively.

For axisymmetric mean flames, we have $\bar{\xi}_{, \theta} \equiv 0$, thus Eq.(15) can be simplified to obtain:

$$
\begin{aligned}
& \bar{A}=2 \pi \int_{r} r d r \frac{1}{\sin \psi(r)} \\
& \hat{A}^{\prime}=\sum_{m=-\infty}^{\infty} \int_{\theta=0}^{\theta=2 \pi} e^{i m \theta} d \theta \int_{r} r d r \cos \psi(r) \frac{\partial \hat{\xi}_{m}^{\prime}(r, S t)}{\partial r}
\end{aligned}
$$

Note that for the axisymmetric case, we have $j=m$ in Eq.(12) and this has been used in Eq.(17). Two significant results for a general, axisymmetric flame shape are obtained from this expression. For non-axisymmetric modes $(m \neq 0)$, the integral over $\theta$ is zero, which implies 
that (1) only the axisymmetric $m=0$ mode contributes to the global flame area, and (2) the global flame area fluctuations are independent of the azimuthal velocity, $u_{\theta}$.

Thus, while asymmetric helical modes can introduce substantial wrinkling of the flame front, they actually lead to no fluctuations in flame surface area in axisymmetric flows, a result that has experimental support [4].

\section{Non-axisymmetric Mean Flame Effects}

This section generalizes the axisymmetric flame analysis to the case for non-axisymmetric mean flames. It considers the influence of non-axisymmetry on the sensitivity of the global flame response to helical disturbances. First we consider the case of the mean flame nonaxisymmetries being small distortions of a nominally axisymmetric flow/flame - in this case, direct analytical results can be obtained that provides insight into the more general solution characteristics. Then we present numerical results that apply to strong flame shape/flow distortions.

\subsection{Weak Non-axisymmetries}

For the case of weak non-axisymmetries, parameterized by the small parameter, $\eta$, analytically tractable results can be obtained using standard perturbation approaches. Decompose the flowfield and flame position as:

$$
\begin{aligned}
& u_{i}(r, \theta, t)=\bar{U}_{i}(r)+\eta \bar{U}_{\eta, i}(r, \theta)+\varepsilon u_{i}^{\prime}(r, \theta, t) \\
& \xi(r, \theta, t)=\bar{\xi}_{0}(r)+\eta \bar{\xi}_{\eta}(r, \theta)+\varepsilon \xi^{\prime}(r, \theta, t)
\end{aligned}
$$

The flame position fluctuations (in frequency domain) are then decomposed as: 


$$
\hat{\xi}^{\prime}(\omega, r, \theta)=\hat{\xi}_{0}^{\prime}(\omega, r, \theta)+\eta \hat{\xi}_{\eta}^{\prime}(\omega, r, \theta)
$$

The perfectly axisymmetric $\eta=0$ flame surface is governed by the equation:

$$
\bar{U}_{z}-\bar{U}_{r} \frac{d \bar{\xi}_{0}}{d r}=s_{L} \sqrt{1+\left(\frac{d \bar{\xi}_{0}}{d r}\right)^{2}}
$$

Then, for $\eta \neq 0$, the leading order non-axisymmetric "correction" to the mean flame is governed by:

$$
\left(\overrightarrow{\bar{U}}_{t} \cdot \vec{\nabla}\right) \bar{\xi}_{\eta}=\bar{U}_{\eta, z}-\bar{U}_{\eta, r} \frac{\partial \bar{\xi}_{0}(r)}{\partial r}=\frac{\bar{U}_{\eta, n}(r, \theta)}{\sin \psi(r)}
$$

Where the subscript $n$ corresponds to the normal component to the axisymmetric mean flame. Thus, the mean flow asymmetries normal to the perfectly axisymmetric part of the mean flame will drive the non-axisymmetries in the mean flame surface. Similarly, the flame wrinkling can decomposed as:

$$
\begin{gathered}
i 2 \pi S t \hat{\xi}_{0}^{\prime}+\left(\overrightarrow{\bar{U}}_{t} \cdot \vec{\nabla}\right) \hat{\xi}_{0}^{\prime}=\hat{u}_{z}^{\prime}-\hat{u}_{r}^{\prime} \frac{\partial \bar{\xi}_{0}(r)}{\partial r} \\
i 2 \pi S t \hat{\xi}_{\eta}^{\prime}+\left(\overrightarrow{\bar{U}}_{t} \cdot \vec{\nabla}\right) \hat{\xi}_{\eta}^{\prime}=-\left[\left(\overrightarrow{\hat{u}}^{\prime} \cdot \vec{\nabla}\right) \bar{\xi}_{\eta}+\left(\overrightarrow{\bar{U}}_{\eta} \cdot \vec{\nabla}\right) \hat{\xi}_{0}^{\prime}+s_{L} \sin (\psi(r))\left[\vec{\nabla} \bar{\xi}_{\eta} \cdot \vec{\nabla} \hat{\xi}_{0}^{\prime}\right]\right]_{(24)}
\end{gathered}
$$

Equation (23) indicates that the flow fluctuations directly lead to the leading order flame wrinkling (corresponds to wrinkling when $\eta=0$ ) However there are multiple sources of disturbances for the additional "corrected" flame wrinkling governed by Eq.(24): (1) interaction of mean flame non-axisymmetries with flow disturbances, (2) interaction of mean flow nonaxisymmetries with the leading order local flame wrinkling, and (3) wrinkle convection along the non-axisymmetric mean flame direction. Note that all three terms consist of the interaction of asymmetric helical modes in the disturbance flow with the non-axisymmetries in the mean flame. 
We will illustrate explicit solutions to these expressions in the next section.

It has been shown earlier for Eq.(23) that only the axisymmetric component of the RHS, i.e. the $m=0$ mode of the RHS (flow fluctuations in this case) contributes to the global FTF. This result applies to Eq.(24) as well, wherein only the $m=0$ mode in the RHS contributes to the global FTF. The RHS of Eq.(24) comprises the product of terms, each of which are azimuthally varying, and thus the co- and corresponding counter- non-axisymmetric modes of each sub-term can multiply to give axisymmetric contributions. Thus for non-axisymmetric mean flames, the non-axisymmetric helical modes can lead to nonzero global heat release, unlike axisymmetric mean flames where only the axisymmetric mode leads to a global flame response. This is better explained using an example problem presented later.

\subsection{Strong Non-axisymmetries}

For the case of strong non-axisymmetries in the mean flame/flow, analytical treatments are not possible due to the highly non-linear nature of Eq.(4) and thus, the solutions are obtained using numerical methods. The spatial derivatives are discretized using a Weighted Essentially NonOscillatory (WENO) [51] scheme designed for Hamilton-Jacobi equations. This scheme is uniformly fifth order accurate in regions where the spatial gradients are smooth and third order accurate in discontinuous regions. Derivatives at the boundary nodes are calculated using fifth order accurate upwind-differencing schemes so that only the nodes inside the computational domain were utilized. A Total Variation Diminishing (TVD) Runge-Kutta scheme [52], up to third order accurate, was used for time integration and Local Lax-Friedrich (LLF) scheme, was used for improved stability [51]. 
The spatial grid is chosen based on resolving $1 / 100^{\text {th }}$ of the smallest length scale and the time-step is chosen to capture at least $1 / 1000^{\text {th }}$ of the time-period of the forcing frequency. Note that the spatial grid is two-dimensional in the radial and azimuthal directions. A typical radialazimuthal grid ranges between 500x1000 to $1000 \times 1000$ grid points, which was chosen based on the grid independence of the unsteady global heat release rate time-series. The numerical solver was developed in the $\mathrm{C}$ language using OpenMP for parallel computing.

\section{Example Calculations}

\subsection{Weak Mean Flame Non-axisymmetries}

In this section we consider example calculations to explicitly illustrate the general conclusions presented earlier. Consider the following non-axisymmetric flow field:

$$
\begin{aligned}
& \bar{U}_{r}=0 \\
& \bar{U}_{\theta}=2 \pi S t(\Omega / \omega) r=2 \pi S t \sigma r \\
& \bar{U}_{z}=1+\eta \bar{U}_{\eta, z}(\theta)
\end{aligned}
$$

Here, $\Omega r$ is the solid-body rotation flow field in the swirl direction (where $\Omega$ is the rate of rotation) and $\sigma=\Omega / \omega$ is a non-dimensional ratio of the acoustic time-scale and swirling timescale. The mean flow non-axisymmetries are considered only in the axial flow for illustrative purposes. The non-axisymmetric components of the mean flow can be brought forward explicitly as:

$\bar{U}_{\eta, z}(r, \theta)=A_{\eta, z, 0}^{u}(r)+\sum_{n \geq 0}\left[A_{\eta, z, n}^{u}(r) \cos n \theta+B_{\eta, z, n}^{u}(r) \sin n \theta\right]$

From Eq.(21), we have: 
$\bar{\xi}_{0}(r)=(r-R) \cot \psi=\tilde{r}$

The solution to Eq.(22) is given by:

$\bar{\xi}_{\eta}(\tilde{r}, \theta)=A_{\eta, z, 0}^{\xi}(\tilde{r})+\sum_{n \geq 0}\left[A_{\eta, z, n}^{\xi}(\tilde{r}) \cos n \theta+B_{\eta, z, n}^{\xi}(\tilde{r}) \sin n \theta\right]$

Here,

$$
\begin{aligned}
& A_{\eta, z, 0}^{\xi}(\tilde{r})=\frac{1}{\cos ^{2} \psi_{0}} \int_{s=0}^{s=\tilde{r}} A_{\eta, z, 0}^{u}(f(s)) d s \\
& \frac{A_{\eta, z, n}^{\xi}(\tilde{r})}{\sec ^{2} \psi_{0}}=\int_{s=0}^{s=\tilde{r}}\left[A_{\eta, z, n}^{u}(f(s)) \cos \left(n S t_{\Omega, 2}(s-\tilde{r})\right)+B_{\eta, z, n}^{u}(f(s)) \sin \left(n S t_{\Omega, 2}(s-\tilde{r})\right)\right] d s \\
& \frac{B_{\eta, z, n}^{\xi}(\tilde{r})}{\sec ^{2} \psi_{0}}=\int_{s=0}^{s=\tilde{r}}\left[B_{\eta, z, n}^{u}(f(s)) \cos \left(n S t_{\Omega, 2}(s-\tilde{r})\right)-A_{\eta, z, n}^{u}(f(s)) \sin \left(n S t_{\Omega, 2}(s-\tilde{r})\right)\right] d s
\end{aligned}
$$

where $f(s)=R+s \tan \psi_{0}$ and $S t_{\Omega, 2}=S t_{\Omega} / \cos ^{2} \psi_{0}$. Now consider an axially convected, helical disturbance of the form:

$$
\left.\hat{u}_{z, m}^{\prime}(\tilde{r}, \theta)\right|_{\text {flame }}=\exp \left(-\frac{i 2 \pi \mathrm{St}}{k_{c}} \tilde{r}+i m \theta\right)
$$

Where $\tilde{r}=\cot \psi(r-R)$. The leading order local flame wrinkling solution for Eq.(23) is given by:

$$
\hat{\xi}_{0, m}^{\prime}(\tilde{r}, \theta)=\frac{1}{\cos ^{2} \psi}\left[\frac{\sin \left(\pi \chi_{L} \tilde{r}\right)}{\left(\pi \chi_{L}\right)}\right] e^{-i 2 \pi S t_{2} \tilde{r}-i 2 \pi m S t_{\Omega, 2} \tilde{r}+i \pi \chi_{L} \tilde{r}}
$$

Where 


$$
\begin{aligned}
& \chi_{L}=S t_{c}-S t_{2}-m S t_{\Omega, 2} \\
& S t_{2}=\frac{S t}{\cos ^{2} \psi}=\frac{f L_{f}}{U_{0} \cos ^{2} \psi}=\frac{f L_{f}}{\bar{U}_{t, z}} \\
& 2 \pi S t_{\Omega, 2}=S t_{\Omega} / \cos ^{2} \psi=\Omega L_{f} / \bar{U}_{t, z}
\end{aligned}
$$

In order to determine the correction to the leading order flame wrinkling behavior, Eq.(24) must be solved using Eqs.(31)-(33) as inputs. To do this, we need to evaluate the RHS term. Consider the interaction of the $n$-th mode non-axisymmetry in the mean flame shape (Eq.(28)) and the $m$-th mode flame response in the leading order flame wrinkling (Eq.(33)). We shall denote this interaction by $R H S_{n, m}$. We can rewrite Eq.(28) in the form:

$$
\bar{\xi}_{\eta}(\tilde{r}, \theta)=A_{\eta, z, 0}^{\xi}(r)+\frac{1}{2} \sum_{n \neq 0}\left[A_{\eta, z, n}^{\xi}(r)+\operatorname{sign}(n) i B_{\eta, z, n}^{\xi}(r)\right] e^{i n \theta}
$$

Using this, we have:

$$
\frac{R H S_{n, m}}{\sin ^{2} \psi_{0}}=-e^{i(n+m) \theta}\left(\frac{d}{d r}\left[\left(A_{\eta, z, n}^{\xi}(r)-i B_{\eta, z, n}^{\xi}(r)\right)\right] \frac{d \hat{\xi}_{0, m}^{\prime}(\tilde{r})}{d r}-\frac{n m}{r^{2}}\left[\left(A_{\eta, z, n}^{\xi}(r)-i B_{\eta, z, n}^{\xi}(r)\right) \hat{\xi}_{0, m}^{\prime}(\tilde{r})\right]\right)
$$

The resulting flame response correction due to this forcing function can be expressed as $\hat{\xi}_{\eta, n, m}^{\prime}$, where (from Eq.(24)):

$$
i 2 \pi S t \hat{\xi}_{\eta, n, m}^{\prime}+\left(\overrightarrow{\vec{U}}_{t}(r) \cdot \vec{\nabla}\right) \hat{\xi}_{\eta, n, m}^{\prime}=R H S_{n, m}
$$

Note that this is equivalent to a system where the forcing function has a combined azimuthal mode number $(n+m)$. Hence, for cases where $n+m=0$, the global response is non-zero. This implies that the non-axisymmetries in the mean flow $(n \neq 0)$ can interact with helical modes in the disturbance field $(m \neq 0)$ leading to net global flame response, as long as $n+m=0$. The 
importance of a particular helical mode is dictated by the presence of a corresponding mode of opposite sign in the non-axisymmetric mean flame/flow.

For the purposes of illustration, consider an example mean flow field where the nonaxisymmetries are given by:

$$
\begin{aligned}
& A_{\eta, z, 0}^{u}=0 \\
& A_{\eta, z, n}^{u}=B_{\eta, z, n}^{u}=1
\end{aligned}
$$

From a global flame response perspective, only the cases where $n+m=0$ are significant. Hence, we first consider the non-axisymmetric modes $n=-m$ in Eq.(31):

$$
\begin{aligned}
& A_{\eta, z,-m}^{\xi}(\tilde{r})=\frac{1+\sqrt{2} \sin \left(m S t_{\Omega, 2} \tilde{r}-\pi / 4\right)}{m S t_{\Omega}} \\
& B_{\eta, z,-m}^{\xi}(\tilde{r})=\frac{\sqrt{2} \sin \left(m S t_{\Omega, 2} \tilde{r}+\pi / 4\right)-1}{m S t_{\Omega}}
\end{aligned}
$$

Using Eq.(33), Eq.(36) and Eq.(39) in Eq.(37), we have the solution:

$$
e^{i 2 \pi S t_{2} \tilde{r}} \hat{\xi}_{\eta,-m, m}^{\prime}(\tilde{r})=I_{1}+I_{2}+\frac{i m \tan ^{2} \psi_{0}}{4 \pi S t_{\Omega} \chi_{L}}\left(I_{3}+I_{4}+I_{5}+I_{6}\right)
$$

The terms $I_{1-6}$ are defined in the Appendix.

Using these solutions for weak non-axisymmetries, we apply them to example flames shapes. We will specifically consider three examples, motivated by applications, where the dominant amplitude values in the decomposition shown in Eq.(28) are $|n|=1,2$ and 4 respectively. First, we consider an axisymmetric flame whose centerline is displaced from the centerline of the unsteady flow disturbances. As we will show, this leads to nonzero $|n|=1$ modes in the flow disturbance coordinate system (see Figure 5). Then, we consider two examples where the flame 
and flow centerlines are aligned, but the flame is truly non-axisymmetric: (i) an elliptical crosssection (Figure 7), leading to $|n|=2$, and (iii) squircle cross-section (Figure 9), leading to $|n|=4$. For strong non-axisymmetries, the numerical solution procedure described earlier is used. The effects of mean flame non-axisymmetry are understood by comparing the Flame Transfer Function $(F T F)$ which in these examples is defined as:

$$
F T F=\frac{\hat{A}^{\prime} / \bar{A}}{\varepsilon}
$$

\subsection{Strong Mean Flame Non-axisymmetries}

\subsubsection{Offset Flame}

In this section, we consider the situation where the flow disturbances and mean flame centerlines are not aligned. This offset is routinely observed in experiments and grows with downstream distance. This offset can be written as:

$\bar{\xi}(\tilde{r}, \theta)=\bar{\xi}_{0}(\tilde{r})[1+\eta \cos \theta]$

For small $\eta$, the mean flame shape is offset and nearly axisymmetric, but its shape becomes

distorted from axisymmetric for large $\eta$ values. Here, $\bar{\xi}_{0}(\tilde{r})=\tilde{r}$ from Eq.(27). The azimuthal variation in position is shown in Figure 5 for different values of the non-axisymmetry parameter $\eta$, with $\eta=0$ corresponding to the axisymmetric mean flame given by Eq.(27). For the case of weak non-axisymmetries, the mean flame shape correction can be expressed as (using Taylor expansion to first order):

$\bar{\xi}_{\eta}(r, \theta)=\tilde{r} \cos \theta$ 
Hence, we have:

$$
\begin{aligned}
& A_{\eta, z, n=1}^{\xi}(\tilde{r})=\tilde{r} \\
& A_{\eta, z, n \neq 1}^{\xi}(\tilde{r})=B_{\eta, z, n}^{\xi}(\tilde{r})=0 \quad \forall n \geq 0
\end{aligned}
$$

The mean flow field is given by:

$$
\bar{U}_{\eta, z}(r, \theta)=\left[\cot \psi \cos \theta-S t_{\Omega} \tilde{r} \sin \theta\right]
$$

This results in the following non-axisymmetric mode coefficients:

$$
\begin{aligned}
& A_{\eta, z, n=1}^{u}(\tilde{r})=\cot \psi \\
& B_{\eta, z, n=1}^{u}(\tilde{r})=-S t_{\Omega} \tilde{r} \\
& A_{\eta, z, n \neq 1}^{u}(\tilde{r})=B_{\eta, z, n \neq 1}^{u}(\tilde{r})=0 \quad \forall n \geq 0
\end{aligned}
$$

These equations show that for a weakly non-axisymmetry flame with an offset cross-section, there exists only the modes: $n=0, \pm 1$. The $n= \pm 1$ mode in the mean flow/flame nonaxisymmetry interacts with the helical modes $m=\mp 1$ in the flow disturbance, leading to a finite global flame response. For the example calculations presented next, we consider the cases: (i) effect of $\eta$ on the FTF amplitude and (ii) effect of swirl ( $\left.S t_{\Omega}\right)$ on the FTF amplitude.

Figure 6(a) illustrates the effect of degree of non-axisymmetry, parameterized by $\eta$, on the FTF amplitude of a flame excited by a $m=1$ helical mode. As seen in the figure, for low values of $\eta$, the FTF amplitude asymptotes to zero and increases with $\eta$. For values of $\eta<0.25$, there is a good match between the FTF obtained using the numerical solver and that obtained using the asymptotic solution shown in Eq.(40).

Next, consider the effect of Strouhal number and swirl on the FTF amplitude. This is shown in Figure 6(b) for the offset flame with $\eta=0.3$. The solid curves denote the response of this non- 
axisymmetric flame to a symmetric flow disturbance $(m=0)$. Note that the low Strouhal number transfer function begins from unity and then decreases with increasing Strouhal number. In the case of perfectly axisymmetric mean flames, the swirl component has no effect on the global FTF amplitude. This is not true, however, for the case of non-axisymmetric mean flames as shown by the comparison of the $F T F$ amplitude for different values of $S t_{\Omega}$. As the value of $S t_{2}$ is increased, the $F T F$ amplitudes for the different $S t_{\Omega}$ cases deviate from each other. This can be attributed to the interference effects introduced by the transport of wrinkles on the nonaxisymmetric flame surface by the swirling flow. In the example considered here, only the response to the $m=1$ mode has been presented. Note that the response to the $m=-1$ helical mode is also non-zero (because both $n=1$ and $n=-1$ values are present in the mean flame position), and that this response is quantitatively different from the $m=1$ mode since the flow is swirling in the anti-clockwise direction.

Even while the transfer function associated with the $m=1$ mode is substantially lower than the $m=0$ transfer function, this does not imply that the heat release response of the flame to $m=1$ helical disturbances is negligible relative to the axisymmetric, $m=0$ mode. The unsteady heat release rate oscillation is directly proportional to the product of the $F T F$ and the amplitude of the velocity disturbance. For example, considering the helical mode amplitude data shown in Figure 3 , we can clearly see that the $m= \pm 1$ mode has a velocity amplitude that is about 5 times that of the symmetric $m=0$ mode. This implies that even if the symmetric mode $F T F$ amplitude is higher than that of the helical mode FTF amplitude, the unsteady heat release rate oscillations due to both of them are comparable. Thus, a smaller FTF amplitude does not necessarily imply a smaller contribution to the net global unsteady heat release rate in flows with dominant helical modes. 


\subsubsection{Elliptic Cross-section}

In this example, we consider an elliptical flame shape, given by:

$\bar{\xi}(\tilde{r}, \theta)=\bar{\xi}_{0}(\tilde{r}) \sqrt{1-\eta \cos ^{2} \theta}$

Here, $\bar{\xi}_{0}(\tilde{r})=\tilde{r}$ from Eq.(27). The azimuthal variation in position is shown in Figure 7 for different values of the non-axisymmetry parameter $\eta$. Note that for $\eta=0$ we retrieve the axisymmetric mean flame given by Eq.(27). As this value is increased, the cross-section eccentricity increases. For the case of weak non-axisymmetries, the mean flame shape correction can be expressed as:

$$
\bar{\xi}_{\eta}(r, \theta)=\tilde{r}\left(-\frac{1}{4}-\frac{1}{4} \cos 2 \theta\right)
$$

Hence, we have:

$$
\begin{aligned}
& A_{\eta, z, 0}^{\xi}(\tilde{r})=A_{\eta, z, n=2}^{\xi}(\tilde{r})=-\frac{1}{4} \tilde{r} \\
& A_{\eta, z, n \neq 0,2}^{\xi}(\tilde{r})=B_{\eta, z, n}^{\xi}(\tilde{r})=0 \quad \forall n \geq 0
\end{aligned}
$$

The mean flow field is given by:

$$
\bar{U}_{\eta, z}(r, \theta)=\left[-\left(\frac{\cos ^{2} \psi}{4}\right)-\left(\frac{\cos ^{2} \psi}{4}\right) \cos 2 \theta+S t_{\Omega}\left(\frac{\tilde{r}}{2}\right) \sin 2 \theta\right]
$$

This results in the following non-axisymmetric mode coefficients:

$$
\begin{aligned}
& A_{\eta, z, 0}^{u}(\tilde{r})=A_{\eta, z, n=2}^{u}(\tilde{r})=-\left(\frac{\cos ^{2} \psi}{4}\right) \\
& B_{\eta, z, n=2}^{u}(\tilde{r})=S t_{\Omega} \frac{\tilde{r}}{2} \\
& A_{\eta, z, n \neq 0,2}^{u}(\tilde{r})=B_{\eta, z, n \neq 2}^{u}(\tilde{r})=0 \quad \forall n \geq 0
\end{aligned}
$$


Thus the only non-axisymmetric modes here are: $n= \pm 2$. However, for a symmetric mode $m=0$ in the disturbance field, the global flame response is corrected due to the $n=0$ mode in the mean non-axisymmetry. The $n= \pm 2$ mode in the mean flow/flame non-axisymmetry interacts with the helical modes $m=\mp 2$, as shown in Figure 8 .

The qualitative nature of the $F T F$ curves is similar to that of the previous example. However note that the response to the helical modes is relatively more significant and is comparable to the symmetric mode response at lower frequencies when compared to the offset flame case.

\subsubsection{Squircle Cross-section}

In this final example, we consider calculations for a flame whose mean shape cross-section is a "squircle" (square-circle, i.e. a square with rounded corners), given by:

$\bar{\xi}(\tilde{r}, \theta)=\bar{\xi}_{0}(\tilde{r})\left[|\cos \theta|^{2+\eta}+|\sin \theta|^{2+\eta}\right]^{1 /(2+\eta)}$

Here, $\bar{\xi}_{0}(\tilde{r})=\tilde{r}$ from Eq.(27). The cross-section shape variations are depicted in Figure 9(a). Note that this approaches a true circle and square for $\eta=0$ and $\eta \rightarrow \infty$, respectively. This shape was motivated by the experimental data [40] for the center flame image in Figure 2. A representative non-axisymmetric mean flame shape is shown in Figure 9 for $\eta=2$. The azimuthal periodicity is of order: $n=4$. Note that modes that are multiples of $n=4$ have dominant amplitudes compared to the other modes. The modal coefficients are given by:

$$
\begin{aligned}
& A_{\eta, z, n=0}^{\xi}(\tilde{r})=-0.193 \tilde{r}, A_{\eta, z, n=4}^{\xi}(\tilde{r})=0.167 \tilde{r} \\
& A_{\eta, z, n=8}^{\xi}(\tilde{r})=0.017 \tilde{r}, \quad B_{\eta, z, n}^{\xi}(\tilde{r}) \approx 0.0
\end{aligned}
$$


For a symmetric mode $m=0$ in the disturbance field, the global flame response is corrected due to the $n=0$ mode in the mean non-axisymmetry. The $n= \pm 4$ modes in the mean flow/flame non-axisymmetry interacts with the helical modes $m=\mp 4$ (and its multiples) in the flow disturbance, leading to a finite global flame response. The follow calculations illustrate example results for the $\eta=2 ; m=0,4$ modes.

The solid curves in Figure 10 show the effect of swirl on the FTF amplitude for an axisymmetric flow disturbance $(m=0)$. As the value of $S t_{\Omega}$ is increased, the $F T F$ amplitude shows little difference. In contrast, consider the dashed curves which show the comparison of the FTF amplitude under the presence of a helical flow disturbance $(m=4)$. These results clearly indicate the variation of the $F T F$ amplitude due to different helical modes in the disturbance field for different control parameters. While the qualitative features of the FTF are similar to that of the previous examples, an important feature to consider here is the effect of swirl. Notice that the FTF interference patterns are affected to a greater extent by swirl in the mean flow when compared to the previous examples.

\section{Conclusions}

A key finding from this work is that helical modes influence the FTF of axisymmetric and non-axisymmetric mean flames differently. In the case of axisymmetric mean flames, only the axisymmetric contribution of the flame wrinkling behavior contributes to the global fluctuating area - thus the heat release of the flame is controlled by the strength of the $m=0$ mode, irrespective of the other helical modes in the flow. However, in the case of non-axisymmetric mean flames, helical modes make non-zero contributions to the $F T F$. Depending on the strength of the non-axisymmetry, the flame length, Strouhal number and swirl number, the helical mode 
contribution to the FTF can also be the dominant contribution when compared with the symmetric $(m=0)$ mode contribution. Thus, this paper shows one mechanism through which significantly different sensitivities may be observed in single and multi-nozzle flames in otherwise identical hardware. In particular, this mechanism is operative in flows with strong helical disturbances. The results here allow the analyst to determine the significance that distortions in flame shape have upon the forced response of the flames. Finally, we should emphasize that this paper's attention is focused to weak disturbances - non-linear effects at high perturbation amplitude, in the presence of multiple helical modes, can cause nonzero heat release response for even axisymmetric flames through a triadic interaction mechanism (axisymmetric flames respond with a non-zero $F T F$ in the presence of up to three different asymmetric helical modes) [53].

\section{Acknowledgements}

This work has been partially supported by the US Department of Energy under contract DENT0005054 (contract monitor Mark Freeman), the National Science Foundation through contract CBET-1235779 (contract monitor Prof. Ruey-Hung Chen) and the Air Force Office of Scientific Research through contract \#FA9550-12-1-0107/RC657 (contract monitor Dr. Chiping Li). The numerical simulations were performed on the Nautilus, Blacklight and Stampede cluster systems offered through the National Science Foundation's eXtreme Science Engineering and Discovery Environment (XSEDE) under charge numbers TG-CTS130016 and TG-DMS130001.

\section{Appendix}

The different terms of the solution in Eq.(40) are: 


$$
\begin{aligned}
& I_{1}=\frac{1}{2 i \alpha_{1}}\left(1-\frac{S t_{2}(1+m \sigma)}{\chi_{L}}\right)\left(1-e^{i \alpha_{1} \tilde{r} / \cos ^{2} \psi_{0}}\right) \\
& I_{2}=\frac{1}{2 i \alpha_{2}}\left(\frac{S t_{2}(1+m \sigma)}{\chi_{L}}\right)\left(e^{i \alpha_{1} \tilde{r} / \cos ^{2} \psi_{0}}-1\right) \\
& I_{3}=\int_{0}^{\tilde{r} / \cos ^{2} \psi_{0}}\left[\frac{e^{i\left(\alpha_{3}+\alpha_{4}+\alpha_{5}\right) s}}{\left(R+s \cos \psi_{0} \sin \psi_{0}\right)^{2}}\right] d s \\
& I_{4}=-\int_{0}^{\tilde{r} / \cos ^{2} \psi_{0}}\left[\frac{e^{i\left(\alpha_{5}+\alpha_{3}-\alpha_{4}\right) s}}{\left(R+s \cos \psi_{0} \sin \psi_{0}\right)^{2}}\right] d s \\
& I_{5}=-\int_{0}^{\tilde{r} / \cos ^{2} \psi_{0}}\left[\frac{e^{i\left(\alpha_{5}-\alpha_{3}+\alpha_{4}\right) s}}{\left(R+s \cos \psi_{0} \sin \psi_{0}\right)^{2}}\right] d s \\
& I_{6}=\int_{0}^{\tilde{r} / \cos ^{2} \psi_{0}}\left[\frac{e^{i\left(\alpha_{5}-\alpha_{3}+\alpha_{4}\right) s}}{\left(R+s \cos \psi_{0} \sin \psi_{0}\right)^{2}}\right] d s
\end{aligned}
$$

Where

$$
\begin{aligned}
& \alpha_{0}=-2 \pi S t_{2}(1+m \sigma)+2 \pi S t \sigma m \\
& \alpha_{1}=\left(2 \pi \chi_{L} \cos ^{2} \psi_{0}+\alpha_{0} \cos ^{2} \psi_{0}+2 \pi S t\right) \\
& \alpha_{2}=\left(\alpha_{0} \cos ^{2} \psi_{0}+2 \pi S t\right) \\
& \alpha_{3}=2 \pi S t \sigma m \cos ^{2} \psi_{0} \\
& \alpha_{4}=\pi \chi_{L} \cos ^{2} \psi_{0} \\
& \alpha_{5}=\alpha_{0} \cos ^{2} \psi_{0}-\pi m S t \sigma \cos ^{2} \psi_{0}+\pi \chi_{L} \cos ^{2} \psi_{0}
\end{aligned}
$$

And

$$
\int_{0}^{\tilde{r} / \cos ^{2} \psi_{0}}\left[\frac{\cos \psi_{0} \sin \psi_{0} e^{i \beta s}}{\left(R+s \cos \psi_{0} \sin \psi_{0}\right)^{2}}\right] d s=\left\{\left[\frac{1}{R}-\frac{e^{i \beta \tilde{r} / \cos ^{2} \psi_{0}}}{r}\right]+i \beta e^{\frac{i \beta R}{\cos \psi_{0} \sin \psi_{0}}}\left[\begin{array}{c}
E i\left(\frac{i \beta r}{\cos \psi_{0} \sin \psi_{0}}\right) \\
-E i\left(\frac{i \beta R}{\cos \psi_{0} \sin \psi_{0}}\right)
\end{array}\right]\right\}
$$

$$
E i(x)=-\int_{-x}^{\infty} \frac{e^{-s}}{s} d s
$$




\section{References}

1. T. Lieuwen, Unsteady Combustor Physics, Cambridge University Press, New York, NY, USA, 2012.

2. C. O. Paschereit; E. Gutmark; W. Weisenstein, Excitation of thermoacoustic instabilities by interaction of acoustics and unstable swirling flow, AIAA J. 38 (6) (2000) 1025-1034

3. J. F. Bourgouin; D. Durox; J. P. Moeck; T. Schuller; S. Candel, Self-sustained instabilities in an annular combustor coupled by azimuthal and longitudinal acoustic modes., ASME Turbo Expo (2013).

4. J. P. Moeck; J.-F. Bourgouin; D. Durox; T. Schuller; S. Candel, Nonlinear interaction between a precessing vortex core and acoustic oscillations in a turbulent swirling flame, Combust. Flame 159 (8) (2012) 2650-2668

5. M. Stohr; I. Boxx; C. D. Carter; W. Meier, Experimental study of vortex-flame interaction in a gas turbine model combustor, Combust. Flame 159 (8) (2012) 2636-2469

6. N. Worth; J. Dawson, Self-excited Circumferential Instabilities in a Model Annular Gas Turbine Combustor: Global Flame Dynamics, Proc. Combust. Inst. 34 (2) (2013) 3127-3134

7. N. Worth; J. Dawson, Modal dynamics of self-excited azimuthal instabilities in an annular combustion chamber, Combust. Flame 160 (2013) 2476-2489

8. J. O'Connor; T. Lieuwen, Recirculation Zone Dynamics of a Transversely Excited Swirl Flow and Flame, Phys. Fluids 24 (2012)

9. M. Aguilar; M. Malanoski; G. Adhitya; B. Emerson; V. Acharya; D. Noble; T. Lieuwen, Helical Flow Disturbances in a Multinozzle Combustor, J. Eng. Gas Turb. Power 137 (9) (2015) 091507-091507-9

10. N. Syred, A review of oscillation mechanisms and the role of the precessing vortex core (PVC) in swirl combustion systems, Prog. Energy Combust. Sci. 32 (2) (2006) 93-161

11. C. E. Cala; E. C. Fernandes; M. V. Heitor; S. S.I., Coherent structures in unsteady swirling jet flow, Expt. Fluids 40 (2) (2006) 267-276 10.1007/s00348-005-0066-9.

12. P. Jochmann; A. Sinigersky; M. Hehle; O. Schafer; R. Koch; H. J. Bauer, Numerical simulation of a precessing vortex breakdown, Int. J. Heat Fluid Flow 27 (2) (2006) 192-203 10.1016/j,ijheatfluidflow.2005.08.003.

13. W. Fick; A. J. Griffiths; T. O'Doherty, Visualisation of the Precessing Vortex Core in an unconfined Swirling flow, Opt. Diag. Eng. 2 (1) (1997) 19-31

14. S. I. Shtork; N. F. Vieira; E. C. Fernandes, On the identification of helical instabilities in a reacting swirling flow, Fuel 87 (10-11) (2008) 2314-2321

15. S. Wang; Z. Rusak, The Dynamics of a Swirling Flow in a Pipe and Transition to Axisymmetric Vortex Breakdown, J. Fluid Mech. 340 (1997) 177-223

16. O. Lucca-Negro; T. O'Doherty, Vortex breakdown: a review, Prog. Energy Combust. Sci. 27 (4) (2001) 431-481

17. E. Serre; P. Bontoux, Vortex breakdown in a three-dimensional swirling flow, J. Fluid Mech. 459 (2002) 347-370 10.1017/S0022112002007875.

18. T. Lieuwen; V. McDonell; D. A. Santavicca; T. Sattelmayer, Burner Development and Operability Issues Associated with Steady Flowing Syngas Fired Combustors, Combust. Sci. Tech. 180 (6) (2008) 1169-1192

19. C. Armitage; R. Balachandran; E. Mastorakos; R. Cant, Investigation of the nonlinear response of turbulent premixed flames to imposed inlet velocity oscillations, Combust. Flame 146 (3) (2006) 419-436 

transfer function of a laminar premixed flame, Proc. Combust. Inst. 28 (2000) 765-773

21. A. Dowling, A kinematic model of a ducted flame, J. Fluid Mech. 394 (-1) (1999) 51-72

22. Preetham; H. Santosh; T. Lieuwen, Dynamics of Laminar Premixed Flames Forced by Harmonic Velocity Disturbances, J. Prop. Power 24 (6) (2008) 1390-1402

23. Y. Huang; V. Yang, Effect of swirl on combustion dynamics in a lean-premixed swirlstabilized combustor, Proc. Combust. Inst. 30 (2) (2005) 1775-1782

24. D. Liepmann; M. Gharib, The role of streamwise vorticity in the near-field entrainment of round jets, J. Fluid Mech. 245 (1992) 643-668

25. B. Pier, Local and global instabilities in the wake of a sphere, J. Fluid Mech. 603 (2008) $39-61$

26. M. P. Juniper, The effect of confinement on the stability of non-swirling round jet/wake flows, J. Fluid Mech. 605 (2008) 227-252

27. T. Loiseleux; J. Chomaz; P. Huerre, The effect of swirl on jets and wakes: Linear instability of the Rankine vortex with axial flow, Phys. Fluids 10 (1998) 1120

28. M. P. Juniper, Absolute and convective instability in gas turbine fuel injectors, ASME Turbo Expo (2012).

29. K. Oberleithner; M. Sieber; C. Nayeri; C. Paschereit; C. Petz; H.-C. Hege; B. Noack; I. Wygnanski, Three-dimensional coherent structures in a swirling jet undergoing vortex breakdown: stability analysis and empirical mode construction, J. Fluid Mech. 679 (2011) 383414

30. K. Oberleithner; C. Paschereit; I. Wygnanski, On the impact of swirl on the growth of coherent structures, J. Fluid Mech. 741 (2014) 156-199

31. K. Oberleithner; O. Paschereit, Hydrodynamic instabilities in swirl-stabilized combustion: experimental assessment and theoretical modelling, Bul. Amer. Phys. Soc. 59 (2014)

32. L. Rayleigh, On the stability, or instability, of certain fluid motions, Proc. London Math. Soc. 1 (1) (1879) 57-72

33. L. Rayleigh, On the dynamics of revolving fluids, Proc. Royal Soc. London. Series A (1917) 148-154

34. A. Lacarelle, Faustmann, T., Greenblatt, D., Paschereit, C. O., Lehmann, O., Luchtenburg, D. M., and Noack, B. R., Spatiotemporal Characterization of a Conical Swirler Flow Field Under Strong Forcing, J. Eng. Gas Turb. Power 131 (2009) 031504

35. B. Bellows; M. Bobba; A. Forte; J. Seitzman; T. Lieuwen, Flame transfer function saturation mechanisms in a swirl-stabilized combustor, Proc. Combust. Inst. 31 (2) (2007) 31813188

36. S. K. Thumuluru; T. Lieuwen, Characterization of acoustically forced swirl flame dynamics, Proc. Combust. Inst. 32 (2) (2009) 2893-2900

37. S. K. Thumuluru; M. K. Bobba; T. Lieuwen, Mechanism of the Nonlinear Response of a Swirl Flame to Harmonic Excitation, ASME Turbo Expo (2007).

38. B. Bellows; Y. Neumeier; T. Lieuwen, Forced response of a swirling, premixed flame to flow disturbances, J. Prop. Power 22 (5) (2006) 1075

39. V. Acharya; D. H. Shin; T. Lieuwen, Premixed Flames Excited by Helical Disturbances: Flame Wrinkling and Heat Release Oscillations, J. Prop. Power 29 (6) (2013) 1282-1291 
40. J. Samarasinghe; S. Peluso; M. Szedlmayer; A. DeRosa; B. Quay; D. Santavicca, 3-D Chemiluminescence Imaging of Unforced and Forced Swirl-Stabilized Flames in a Lean Premixed Multi-Nozzle Can Combustor, ASME Turbo Expo (2013).

41. G. Staffelbach; L. Y. M. Gicquel; G. Boudier; T. Poinsot, Large Eddy Simulation of self excited azimuthal modes in annular combustors, Proc. Combust. Inst. 32 (2009) 2909-2916

42. N. Worth; J. Dawson, Tomographic reconstruction of $\mathrm{OH}^{*}$ chemiluminescence in two interacting turbulent flames, Meas. Sci. Tech. 24 (2013)

43. M. Fleifil; A. M. Annaswamy; Z. A. Ghoneim; A. F. Ghoniem, Response of a laminar premixed flame to flow oscillations: A kinematic model and thermoacoustic instability results, Combust. Flame 106 (4) (1996) 487-510

44. G. H. Markstein, Nonsteady Flame Propagation, Pergamon, 1964.

45. M. Matalon; B. Matkowsky, Flames as gasdynamic discontinuities, J. Fluid Mech. 124 (2006) 239-259

46. L. Boyer; J. Quinard, On the Dynamics of Anchored Flames, Combust. Flame 82 (1) (1990) 51-65

47. F. Williams, Turbulent combustion, in: J. Buckmaster (Ed.), The Mathematics of combustion, 1985, pp. 97-131.

48. V. Acharya; Shreekrishna; D. H. Shin; T. Lieuwen, Swirl effects on harmonically excited, premixed flame kinematics, Combust. Flame 159 (2012) 1139-1150

49. P. Palies; T. Schuller; D. Durox; S. Candel, Modeling of premixed swirling flames transfer functions, Proc. Combust. Inst. 33 (2) (2011) 2967-2974

50. H. Y. Wang; C. K. Law; T. Lieuwen, Linear response of stretch-affected premixed flames to flow oscillations, Combust. Flame 156 (4) (2009) 889-895

51. G. Jiang; D. Peng, Weighted ENO Schemes for Hamilton-Jacobi Equations, SIAM J. Sci. Comp. 21 (6) (2000) 2126-2143

52. S. Gottlieb; C. Shu, Total Variation Diminishing Runge-Kutta Schemes, Math. Comput. 67 (221) (1998) 73-85

53. V. Acharya; T. Lieuwen, Premixed Flame Response to Helical Flow Disturbances: Nonlinear Effects, 9th US National Technical Meeting of the Combustion Institute (2015). 


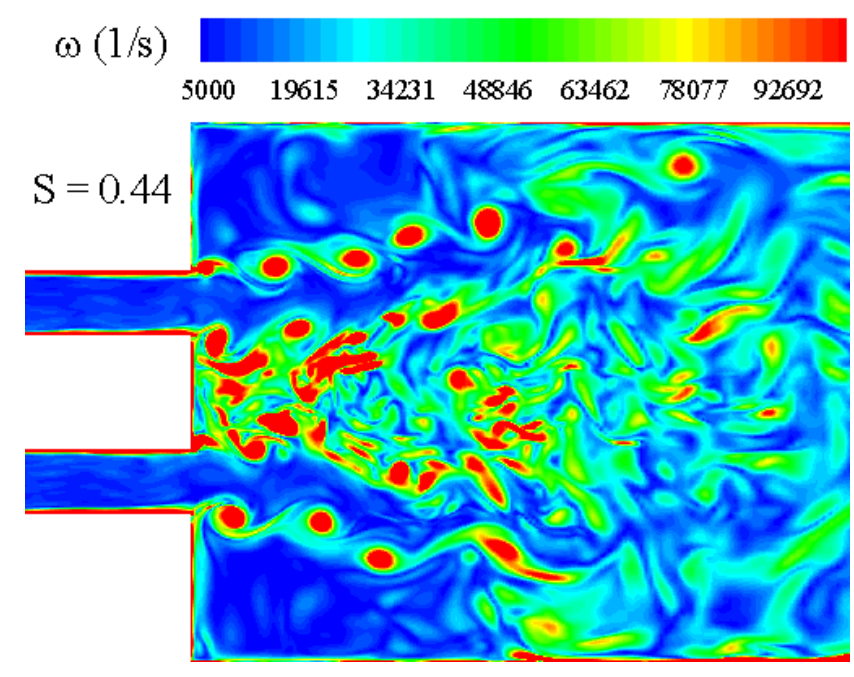

Figure 1 - Instantaneous out of plane vorticity field from LES computations using level-set flame tracking for a swirling flame with flow swirl number of 0.44 . Snapshot courtesy of Prof. Vigor Yang.

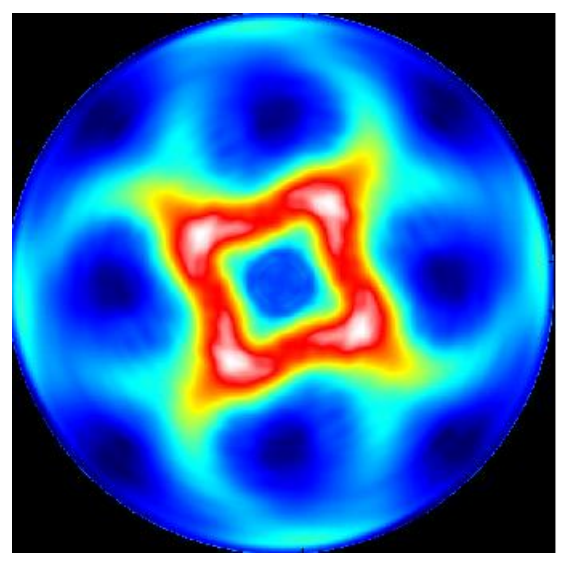

Figure 2 - Heat release intensities from the top view of a 5-flame combustor with flameflame interactions causing a highly non-axisymmetric center flame. Images courtesy of Prof. Domenic Santavicca. 


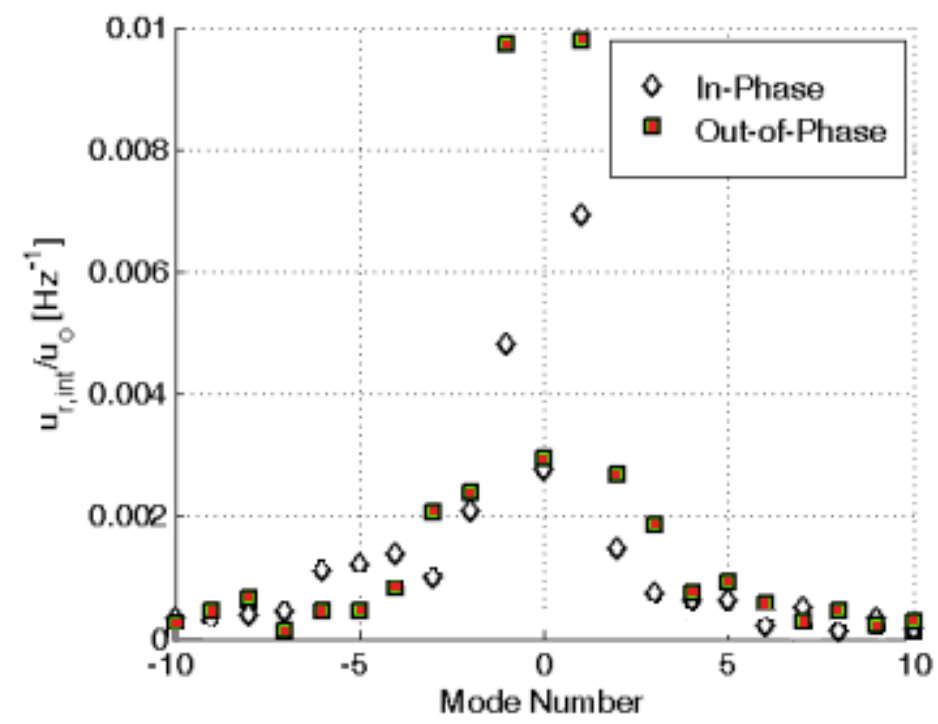

Figure 3 - Amplitude of radial velocity modes for different mode numbers at a fixed location. In-phase (diamonds) and out-of-phase (filled squares) forcing simulate the effect of standing and traveling waves respectively. Plot courtesy Prof. Jacqueline O'Connor.

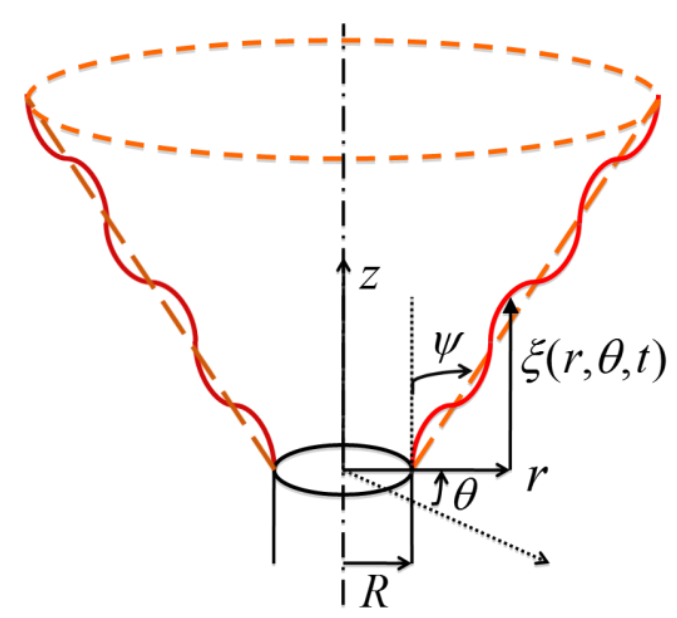

Figure 4 - Flame surface description for the center-body swirl-stabilized premixed flame. 


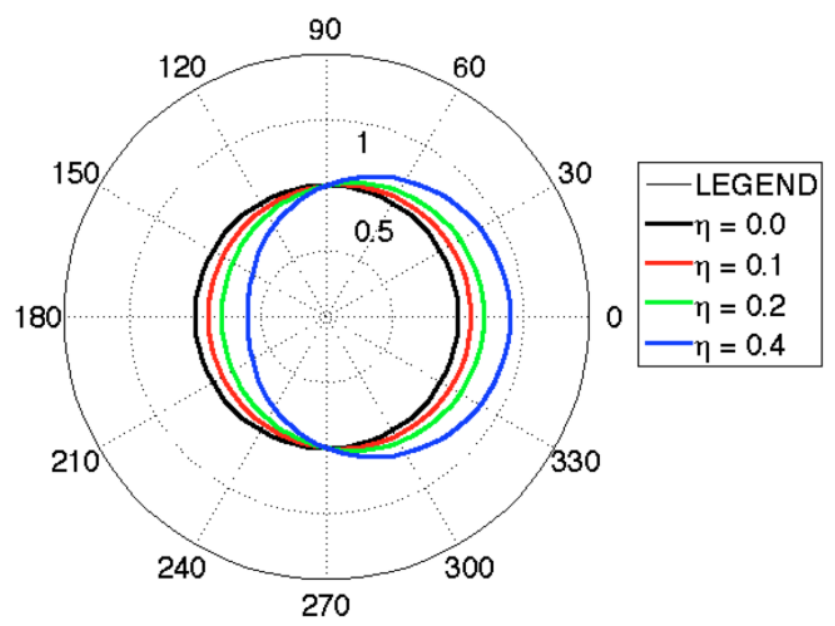

Figure 5 - Offset flame cross-section for varying $\eta$, using Eq.(42).

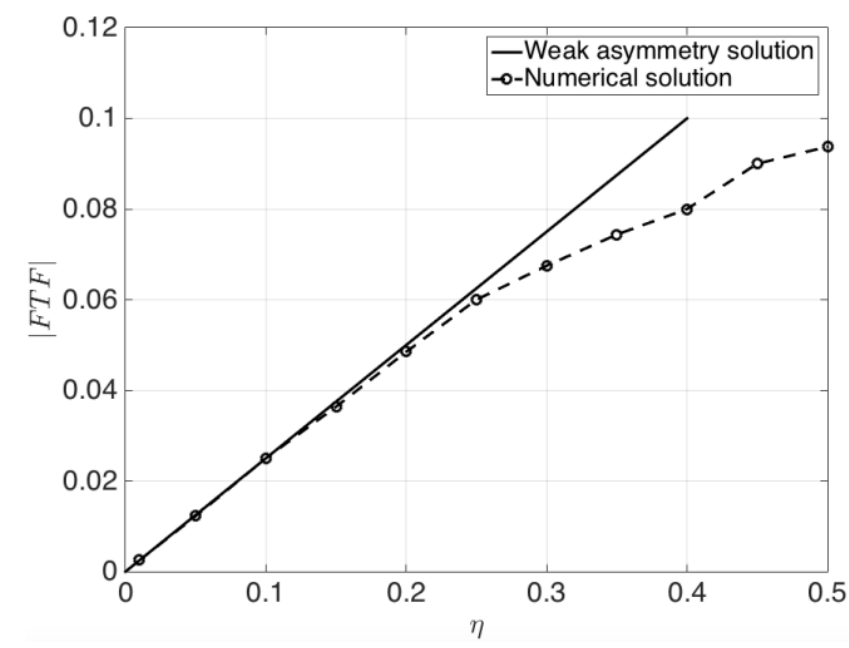

(a)

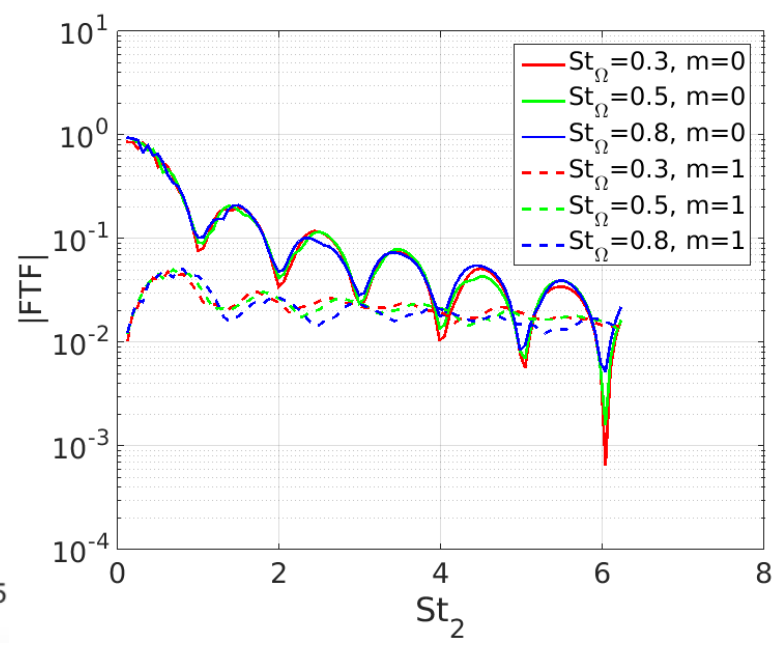

(b)

Figure 6 - Comparison of FTF amplitude for the flame with an offset in its cross-section, (a) For different values of $\eta$ (degree of mean flame offset); (b) For different values of $S t_{\Omega}$ with $\eta=0.3, \psi=20^{\circ}$; in response to a flow disturbance helical mode of $m=1$ and $k_{c}=0.6$. Note that $S t_{2}=f L_{f} / U_{0} \cos ^{2} \psi$. For reference, the response to the symmetric helical mode $(m=0)$ is also shown. 


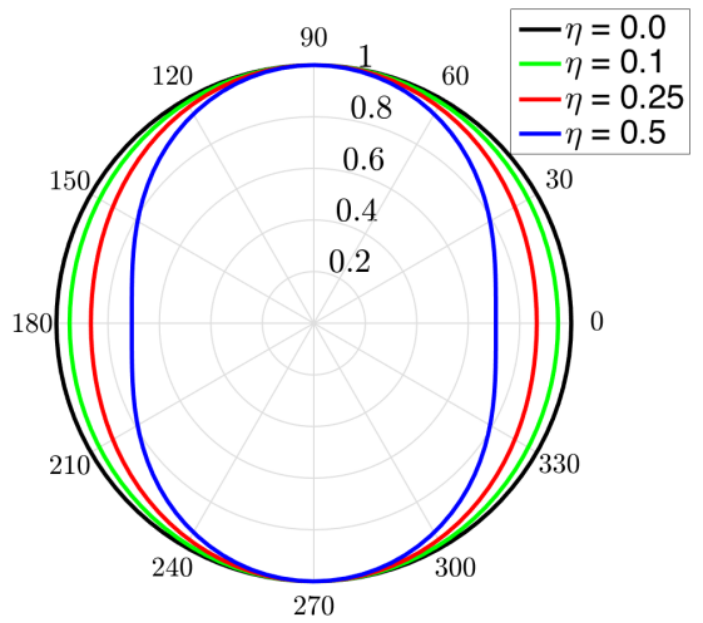

Figure 7 - Elliptic flame cross-section for varying $\eta$, using Eq.(47).

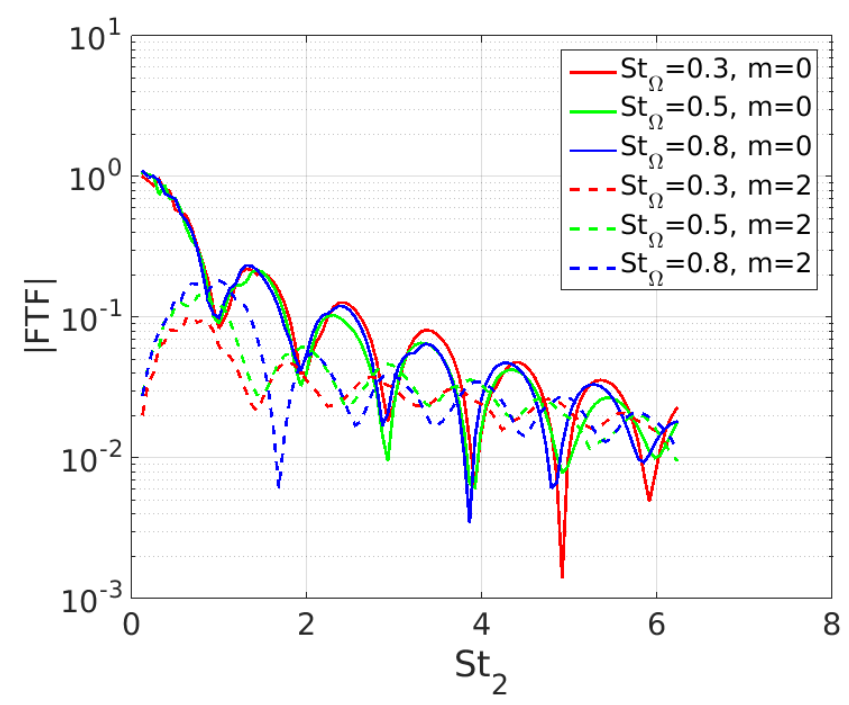

Figure 8 - Comparison of $F$ TF amplitude for an elliptic flame for different values of $S t_{\Omega}$ for an elliptic flame with $\eta=0.5, \psi=20^{\circ}$; in response to a flow disturbance helical mode of $m=2$ and $k_{c}=0.6$. Note that $S t_{2}=f L_{f} / U_{0} \cos ^{2} \psi \cdot$ 
Figure 9 - Squircle cross-section flame surface for $\eta=2$ in Eq.(52) showing isometric view from the side, with surface shading indicating the non-axisymmetry of the mean flame surface.

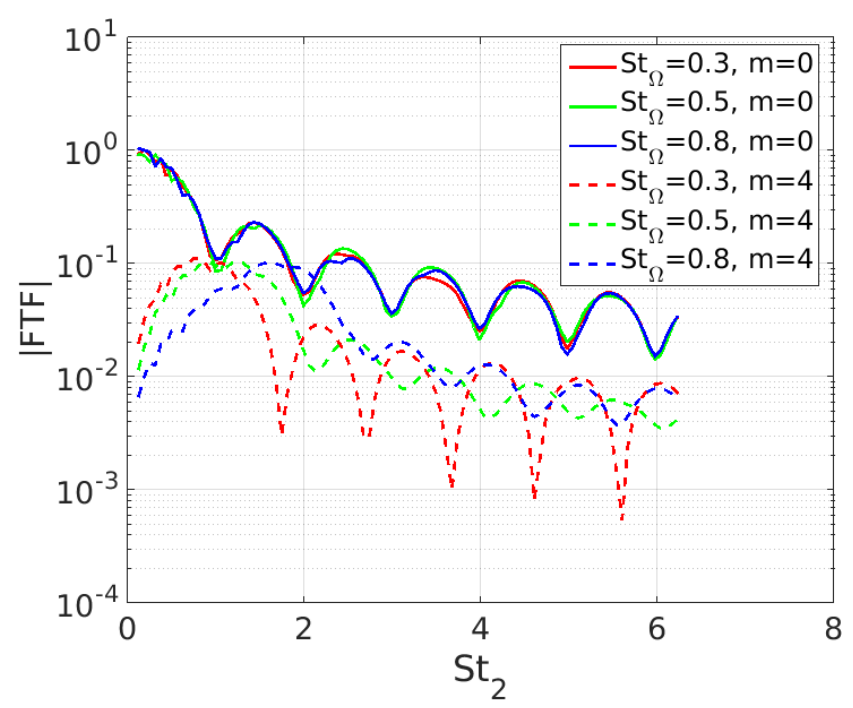

Figure 10 - Comparison of $\boldsymbol{F T F}$ amplitudes for a Squircle cross-section flame responding to a symmetric flow disturbance (solid curves), and responding to helical flow disturbance of mode $m=4$ (dashed curves); for different values of swirl parameter $S t_{\Omega}$ with $k_{c}=0.6$. Note that $S t_{2}=f L_{f} / U_{0} \cos ^{2} \psi$. 